\title{
Metaestabilidad estructural a través de proyección térmica
}

\author{
I.G. CANO, S. DOSTA, J.R. MIGUEL, J.M. GUILEMANY \\ Centro de Proyección Térmica (CPT). Dpt. Ciència dels Materials i Enginyeria Metal.lúrgica. \\ Universitat de Barcelona. C/Martí i Franqués, 1. \\ 08028 Barcelona. Spain
}

\begin{abstract}
Recientes investigaciones en el campo de los materiales cerámicos han dado cuenta de la importancia de la metaestabilidad para obtener estructuras con características singulares. Durante la consolidación del material las fases mestaestables se transforman en una estructura donde se produce la inhibición del crecimiento de grano. Este efecto es una consecuencia directa de la inmiscibilidad de dos fases en estado sólido. Los nanocomposites conseguidos, gracias a su pequeño tamaño de grano y a su estructura uniforme, exhiben unas interesantes propiedades como elevada dureza y tenacidad.

Estas fases metaestables pueden ser producidas por diversas técnicas entre las que se encuentra la proyección térmica. En concreto en este trabajo se ha empleado la Proyección por plasma (APS). Las fases de partida inmiscibles, son fundidas y homogeneizadas durante su corta estancia en la zona caliente del plasma. Seguidamente, las partículas fundidas y aceleradas por el plasma, se someten a un enfriamiento rápido o temple (quenching) en un medio líquido, como el agua o en un substrato enfriado con nitrógeno líquido, formándose a través de este proceso las fases metaestables.

El principal objetivo de este trabajo ha sido la obtención de polvos cerámicos metastables a través de la aplicación de APS y el establecimiento de un proceso de temple conducente a la formación de fases metastables así como la caracterización estructural de éstas. Como última etapa del trabajo se han estudiado los materiales nanoestructurados conseguidos tras realizar tratamientos térmicos.
\end{abstract}

Palabras clave: Metaestabilidad, Proyección térmica, Materiales Nanoestructurados, APS.

Structural metastability through thermal spray.

Recent research in the field of nanostructured ceramic materials has underscored the importance of using feedstock powders with metastable phases. During material consolidation the metastable structure evolves into a dual structure where the grain growth stops when the crystallites reach their respective equilibrium grain size. The mutual suppression of the grain growth is a direct consequence of the immiscibility of the two phases in the solid state. Due to their small grain size and uniform structure, these nanocomposites exhibit very interesting properties such as higher hardness and toughness.

Metastable structures can be produced using Atmospheric Plasma Spray (APS) technique from commercially available micron-sized feedstock powders. The starting immiscible phases become molten and are homogenized during their short residence in the hot zone of the plasma jet. Afterwards, the molten droplets accelerated by the plasma jet are quenched in a liquid medium, such as water, or onto a nitrogen chilled substrate forming metastable phases.

The main target of this work has been the synthesis of metastable ceramic powders through atmospheric plasma spraying (APS) and quenching route. The viability for producing these metastable phases and the structural characterization have been carried out.

Keywords: Metastability, Thermal Spray, Nanostructured Materials, APS.

\section{INTRODUCCIÓN}

Como es bien conocido en el campo de los materiales cerámicos, el sistema Alumina-Titania exhibe propiedades muy interesantes aportando un buen comportamiento al desgaste y a la corrosión así como excelentes propiedades mecánicas como elevada dureza y tenacidad. En las últimas décadas se está trabajando con ahínco en la mejora de propiedades de comportamiento de los materiales a través de la obtención de nanoestructuras que aporten a los materiales características singulares. Un método para favorecer la formación de estas nanoestructuras es utilizar como materiales de partida aquellos constituidos por fases metaestables. Durante la consolidación del material las fases con estructura metaestable evolucionan hacia estructuras binarias, inmiscibles en estado sólido, inhibiendo de este modo el crecimiento de grano (1).

Una vía de obtención de fases metaestables es la proyección térmica, tecnología aplicada habitualmente en la obtención de recubrimientos de diferentes tipos, tanto metálicos como no metálicos y cermets. De hecho, una de las ventajas de los procesos de proyección térmica es la amplia variedad de materiales que pueden ser empleados para obtener recubrimientos $(2,3)$.

El proceso que se ha desarrollado en el Centro de Proyección 
Térmica de la Universidad de Barcelona (CPT) consiste en realizar un enfriamiento rápido (quenching) de las partículas fundidas en la zona caliente del plasma, formándose de este modo fases metaestables. Kear y col. $(4,5)$ han publicado algunos resultados sobre este tema pero poca literatura mas se ha encontrado al respecto.

En concreto, en este trabajo se ha aplicado la tecnología de proyección por plasma para obtener fases con estructura metaestable del sistema $\mathrm{Al}_{2} \mathrm{O}_{3}-\mathrm{TiO}_{2}$ a partir de polvo comercial micrométrico.

\section{EXPERIMENTAL}

El material de partida ha sido polvo de $\mathrm{Al}_{2} \mathrm{O}_{3}-\mathrm{TiO}_{2}$ con distintas características tanto morfológicas como composicionales. En la tabla I se muestran estas materias primas y sus características propias.

El proceso de obtención de fases metaestables se muestra esquemáticamente en la figura 1. Concretamente se ha

TABLA I. CARACTERÍSTICAS DE LOS POLVOS INICIALES UTILIZADOS.

\begin{tabular}{|c|c|c|c|}
\hline \multirow{2}{*}{ Sistema } & $\begin{array}{c}\text { Proceso de } \\
\text { fabricación }\end{array}$ & $\begin{array}{c}\text { Tamaño } \\
\text { de } \\
\text { partícula } \\
(\mu \mathrm{m})\end{array}$ & Fases \\
\hline \multirow{2}{*}{$\mathrm{Al}_{2} \mathrm{O}_{3}-13 w \mathrm{wt} \% \mathrm{TiO}_{2}$} & $\begin{array}{c}\text { Aglomerado y } \\
\text { sinterizado }\end{array}$ & $22.5-45$ & $\alpha-\mathrm{Al}_{2} \mathrm{O}_{3}+$ rutilo- $\mathrm{TiO}_{2}$ \\
\cline { 2 - 4 } & $\begin{array}{c}\text { Fundido y } \\
\text { cuarteado }\end{array}$ & $25-45$ & $\alpha-\mathrm{Al}_{2} \mathrm{O}_{3}+$ anatasa- $\mathrm{TiO}_{2}$ \\
\cline { 2 - 4 } & $\begin{array}{c}\text { Fundido y } \\
\text { cuarteado }\end{array}$ & $10-30$ & $\mathrm{Al}_{2}-\mathrm{Al}_{2} \mathrm{O}_{3}+\mathrm{Al}_{2} \mathrm{TiO}_{5}$ \\
\cline { 2 - 4 } & rutilo- $\mathrm{TiO}_{2}$ \\
\hline
\end{tabular}

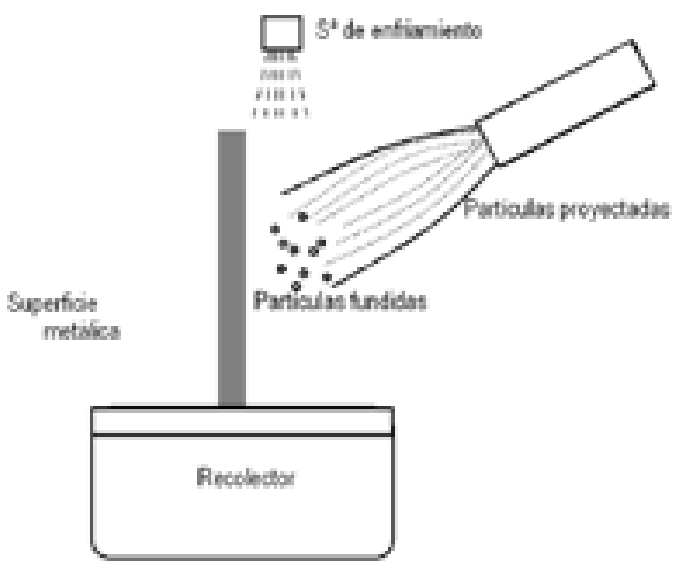

Fig. 1- Esquema del proceso de obtención de fases metaestables a través de proyección térmica + enfriamiento rápido.

utilizado una pistola F4 de plasma y se debe aclarar que se han llevado a cabo diferentes ensayos para comparar resultados, en algunos casos se proyecto el polvo directamente en agua y/o hielo (es decir, en el esquema de la figura 1 se elimino la superficie metálica) y en otros se utilizo una superficie metálica para generar un efecto que se ha denominado de "splat" que produjera una mayor deformación. En este último caso también se comprobó el efecto causado por el tipo de superficie, realizándose pruebas sobre acero y sobre cobre.

Los polvos así obtenidos han sido caracterizados por difracción de rayos $X$, microscopía electrónica de barrido (SEM: JEOL JSM-5310) así como por microscopía de emisión de campo (FESEM: Hitachi H-4100FE ).

Con objeto de valorar la evolución de estas fases metaestables se han realizado tratamientos térmicos a 800 , 1000 y $1200{ }^{\circ} \mathrm{C}$ verificándose las transformaciones de fase y evaluándose la formación de nanoestructuras.

\section{RESULTADOS Y DISCUSIÓN}

Una vez proyectados los polvos iniciales directamente en agua, siguiendo el esquema descrito anteriormente en la figura 1 se ha observado que el polvo presenta una morfología esférica con formación de dendritas. Estas dendritas varían en función del grado de enfriamiento de manera que se ha observado que aparecen zonas de formación dendrítica celular (figura 2a) así como zonas donde aparece una mezcla de estructuras como se puede ver en al figura $2 b$. Estas diferencias son debida a variaciones en la velocidad de enfriamiento durante el proceso y su aparición indica por un lado la necesidad de aumentar la velocidad de enfriamiento para evitar estas formación así como, un mayor control del gradiente de esta velocidad para mejorar la homogeneidad del polvo resultante $(6,7)$.

El polvo así obtenido está compuesto por varias fases: $\mathrm{Al}_{2} \mathrm{O}_{3}$ (fase $\alpha$ ), $\mathrm{TiO}_{2}$ (fase rutile), $\mathrm{Al}_{2} \mathrm{TiO}_{5}$ y $\mathrm{Al}_{2} \mathrm{O}_{3}$ (fase del
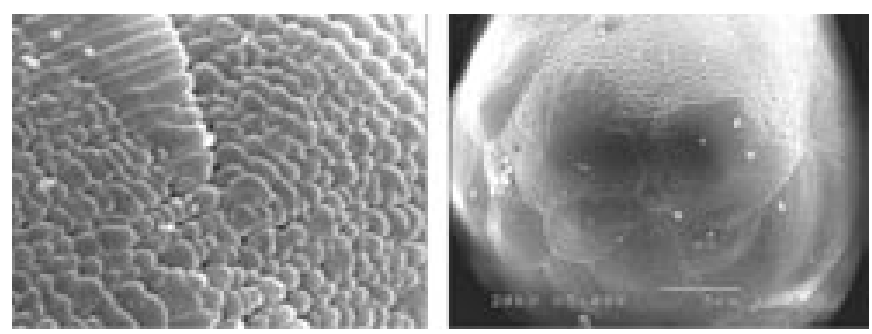

Fig. 2- Micrografias obtenidas por microscopía electrónica de barrido donde se muestra la formación de dendritas en el polvo obtenido (a) dendritas celulares y (b) combinación de formaciones dendríticas.

tipo espinela) como muestra el difractograma de RX de la figura 3. La presencia de rutilo y de corindón prueba que ha existido una separación de fases debido a que la velocidad de enfriamiento no ha sido lo suficientemente rápida. Además la presencia de estas fases, que ya son estables, implica que durante los posibles tratamientos posteriores (procesos de consolidación, tratamientos térmicos, etc.) no transformaran hacia sus formas estables.

Sin embargo, si observamos los resultados obtenidos al proyectar sobre un substrato enfriado encontramos una única fase, $\mathrm{Al}_{2} \mathrm{O}_{3}$ (figura 4). Esta fase corresponde a una estructura cúbica que en la bibliografía se encuentra descrita como tipo espínela donde el Ti ocupa algunos lugares del Al. En la figura 4 también se puede apreciar la comparación de los resultados obtenidos al proyectar sobre agua y sobre otras superficies, acero y cobre concretamente. Es en este último caso donde se han obtenido mejores resultados, produciéndose un polvo compuesto por $\mathrm{Al}_{2} \mathrm{O}_{3}$ tipo espínela y cuya morfología ya no es tan esférica como en el caso de la proyección en agua sino mas deformada y donde las formaciones dendríticas son 


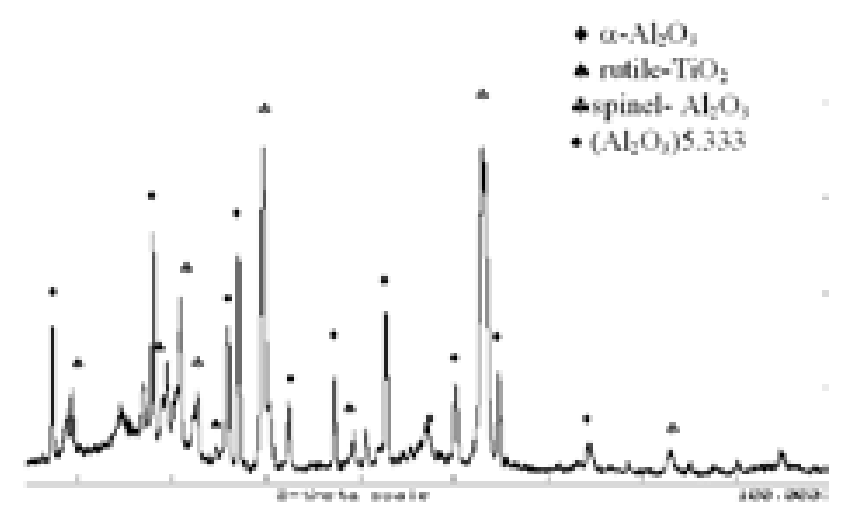

Fig. 3- DRX del polvo obtenido tras su proyección en agua $\left(\mathrm{Al}_{2} \mathrm{O}_{3}\right.$ $13 \%$ en peso de $\mathrm{TiO}_{2}$ ).
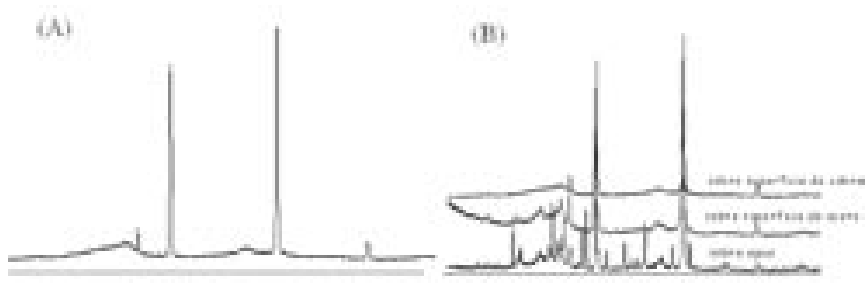

Fig. 4- DRX de los polvos obtenido al proyectar sobre una superficie de cobre enfriada con $\mathrm{N}_{2}$ líquido (A) y comparación de la proyección sobre distintas superficies (B).
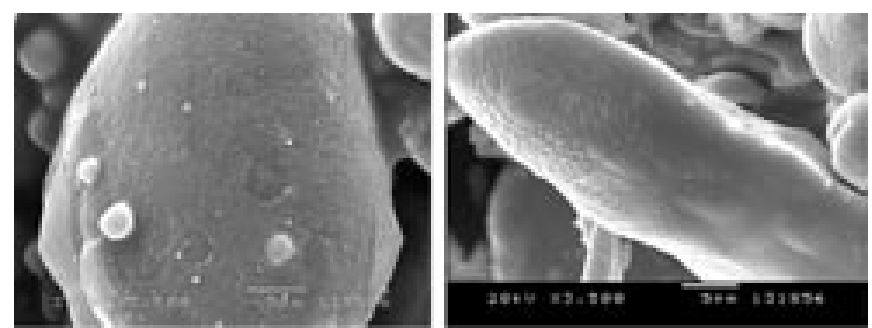

Fig. 5- Micrografías obtenidas por microscopía electrónica de barrido donde se muestra la formación más deformada y con menor segregación del polvo obtenido tras proyectar sobre una superficie de $\mathrm{Cu}$ enfriada.

mas tenues incluso desapareciendo en algunas zonas (figura 5). Esto indica una menor segregación durante el proceso. Además este resultado se ha mantenido independientemente del tipo de polvo inicial, lo que demuestra una correcta fusión y homogeneización del polvo durante la proyección.

Para concluir este trabajo, se han llevado a cabo tratamientos térmicos para evaluar el material resultante tras las correspondientes transformaciones de fase que tienen lugar durante los mismos. La figura 6 muestra los resultados de DRX a diferentes temperaturas, observándose que a $800^{\circ} \mathrm{C}$ existe un considerable desorden estructural con única presencia de la fase de $\mathrm{Al}_{2} \mathrm{O}_{3}$ espinela y trás el tratamiento de $1200^{\circ} \mathrm{C}$ el material ya está compuesto por las dos fases de equilibrio cuya morfología se muestra en la figura 7 donde se puede observar que existen granos de tamaño nanométrico.

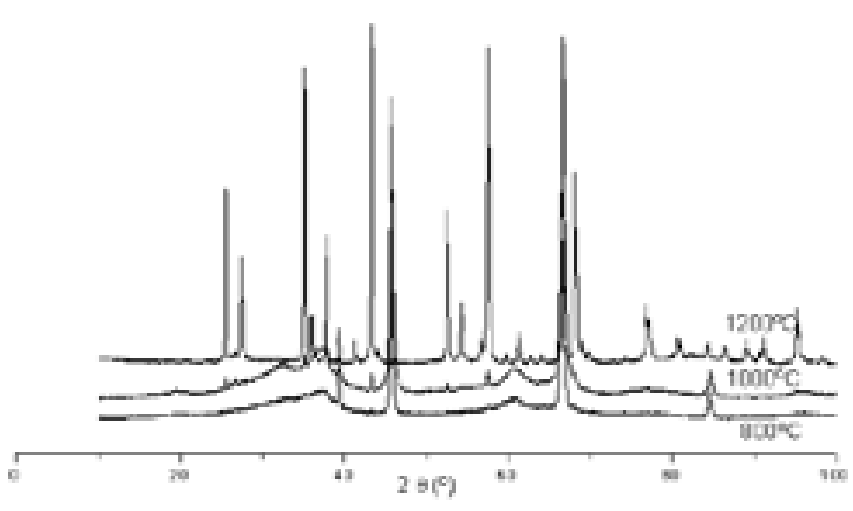

Fig. 6- DRX del material obtenido tras tratamientos térmicos a diferentes temperaturas.
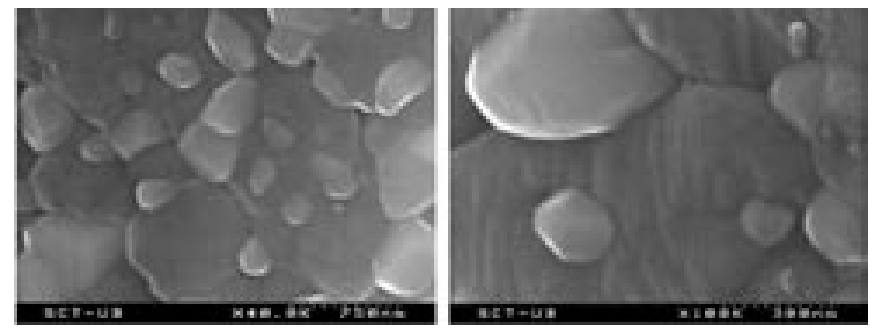

Fig. 7- Micrografías obtenidas por microscopía electrónica de barrido donde se muestra el material obtenido tras un tratamiento térmica a $1200^{\circ} \mathrm{C}$.

\section{CONCLUSIONES}

La composición $\mathrm{Al}_{2} \mathrm{O}_{3}-13 w t \% \mathrm{TiO}_{2}$ ha permitido la obtención de fases metaestables, en concreto $\mathrm{Al}_{2} \mathrm{O}_{3}$ espinela a través de un proceso que combina la proyección con plasma de polvo micrométrico comercial con un enfriamiento rápido en agua y/o nitrógeno líquido.

El tratamiento térmico del polvo así obtenido ha dado lugar a un material nanoestructurado compuesto por $\mathrm{Al}_{2} \mathrm{O}_{3} \mathrm{y}$ precipitados de $\mathrm{TiO}_{2}$

\section{AGRADECIMIENTOS}

Los autores agradecen a la Generalitat de Catalunya el soporte suministrado y a la Unión Europea por su financiación a través del proyecto NMP3-CT-2004-001470

\section{BIBLIOGRAFÍA}

1. S.-C. Liao, W.E. Mayo, K.D. Pae. “Retention of Nanoscale Grain Size in Bulk Sintered Materials Via Pressure-Induced Phase Transformation". Nanostr. Mater., 8 (6), 645-56, (1997)

2. R.Suryanarayanan. "Plasma Spraying: Theory and applications" Ed. World Scientific Publishing Co. Pte. Ltd (1993) London UK.

3. P.Fauchais, M. Vardelle. "Plasma spraying: present and future". Pure \& Appl.Chem., 66 (6), 1247-1258, (1994).

4. B.H. Kear, Z. Kalman, R.K. Sadangi, G. Skandan, J. Colaizzi, W.E. Mayo "Plasma-Sprayed Nanostructured $\mathrm{Al}_{2} / \mathrm{TiO}_{2}$ Powders and Coatings". J. Therm. Spray Tech., 9 (4), 483-487, (2000).

5. B.H. Kear, J. Colaizzi , W.E. Mayo, S.-C. Liao. "On the processing of nanocrystalline and nanocomposites ceramics". Scripta Mater., 44 (8/9), 2065-2068, (2001). 
6. J. Colaizzi, B.H. Kear, W.E. Mayo, R. Shropshire, R.W. Rigney, S. Brunhouse "Micro-and Nano-Scaled Composites via Decomposition of Plasma Sprayed Ceramics". Proceedings of the International Thermal Spray Conference (ITSC), Montreal (Canada), 813-820, (2000).
7. I.G. Cano, S.Dosta, J.R. Miguel and J.M. Guilemany ."Ceramic metastable powder through plasma spraying". Proceedings of International Thermal Spray Conference And Exposition (ITSC) (May 15-18, 2006)

Recibido: $\quad 14.07 .06$

Aceptado: 23.08 .06 